\title{
Laser-intraocular lenses interaction: Aspects to consider for in situ vision correction
}

\author{
G Kareliotis*, E Drakaki, C Bacharis, M Makropoulou and AA Serafetinides \\ Physics Department, School of Applied Mathematical and Physical Sciences, National Technical University of Athens, Zografou Campus, 15780, Athens, Greece
}

\begin{abstract}
Today, 60 years after the laser discovery, the long experience in refractive surgery, the evaluation of the accumulated clinical outcomes and the limitations of keratorefractive surgery generated the demand to develop alternative methods for treating vision defects. In the frame of this new philosophy for vision correction, the use of polymeric intraocular lenses (IOLs) provides sufficient predictability and optical quality. The new Ophthalmology era takes their use one step further, through the IOLs' customization, aiming at the long desired personalized treatment. Inspired by the need to correct some post-operative complications in cataract surgery and IOLs implantation, the laser use is proposed, for in situ refractive modification of the implanted polymeric material, without any alteration in IOL's light transmittance behavior. In this work we present a brief literature overview of new perspectives in this field, enriched with some of our experimental studies. Through discussion of the laser-material interactions we intend to present the prospects, the limitations and the aspects that have to be considered in the route for in situ laser-based IOLsurgery and finally, vision correction.
\end{abstract}

\section{Lasers in ophthalmology and refractive surgery}

During the first laser era, both development and applications of laser radiation were impressive, particularly in life sciences (in all areas: diagnosis, treatment and biomedical research and technology). Just a couple of years after the construction of the first ruby laser by Maiman in 1960, lasers were immediately applied for retinal photocoagulation. Especially in ophthalmology, the laser-based refractive surgery transformed the myopia correction to one of the most effective and rapidly developing eye operations for the general population worldwide. The reader should bear in mind that from the very beginning of laser discovery, the application of laser radiation was easier in ophthalmic tissues, as the human eye is a biophysical optical apparatus which is by its natural assembly customized to accept, transmit and focus ultraviolet, visible and near-infrared (UV, VIS and NIR) photons, in the relevant ocular media, for clear vision. Therefore, the first lasers' biomedical applications were in ophthalmology's field, prior to any attempt of developing light guiding devices (e.g. fibers) to apply laser radiation in internal organs and tissues. A very brief and more or less descriptive review for the first 50 years of ophthalmic laser therapy was reported by Palanker et al. in 2011, as an introductive editorial in Archives of Ophthalmology Journal [1]. Certainly, the early development of laser technology has revolutionized the therapeutical interventions in ophthalmology, mainly in the treatment of the three major causes of blindness: diabetic retinopathy, glaucoma, and agerelated macular degeneration. Categorically, for operation in retina the only non-contact, non-invasive interventional possibility has been the use of VIS or NIR laser light. Since the mid-1980s, the most popular application of lasers in ophthalmology, besides retina, has been corneal refractive surgery, a laser-based surgical method of permanent vision correction by changing eyes' refractive properties.

Today, most refractive disorders are treated with laser ablation techniques in a procedure generally called "laser-based refractive surgery"; although currently, there is a major ongoing effort to refine it in order to correct other vision defects too. For instance, improvements in the development of biocompatible ocular implants used for cataract surgery (e.g. intraocular lenses - IOLs), have provided another option for patients with particular vision defects, besides conventional myopia. Nevertheless, various age-related eye disorders (e.g. cataract, glaucoma, presbyopia, dry-eye syndrome) can influence the possible options for laser-based or non-laser vision correction attempts.

For comprehensiveness reasons, some basic notions of human vision are mentioned. The refractive apparatus of the human eye essentially consists of the cornea, the anterior chamber, the lens and the vitreous. These elements constitute two functional groups for sharp vision, the corneal system and the lens system. The refractive power of the former is approximately 40 to 50 diopters (D), while that of the latter is much lower, approximately 15 to $25 \mathrm{D}$; as measured at accommodative rest with the eyes focused on a distant point [2]. The most common refractive errors, e.g. errors in the ability to focus light by the eye, are:

(a) Myopia, where the eyeball is too long, or the cornea is too steep. In this case, the distance between the cornea and the retina is too long. Hence, light rays from distant objects are focused in the vitreous inside of the eye rather than on the retina, making them look blurry.

(b) Hyperopia, where the cornea's curve is too flat. This makes the cornea - retina distance too short. Light rays from close objects focus behind the retina, making them look blurry.

${ }^{\star}$ Correspondence to: Georgios Kareliotis, Laser Development and Applications Team, Physics Department, National Technical University of Athens, Zografou Campus, 15780, Athens, Greece, E-mail: gkarel@central.ntua.gr

Key words: intraocular lenses, refractive index modification, laser-polymers interaction; IOL customization

Received: June 09, 2020; Accepted: July 17, 2020; Published: July 24, 2020 
(c) Astigmatism, where the cornea is oval shaped instead of rotund, due to an irregular or toric curvature, making both distant and near objects look distorted.

(d) Presbyopia, where the flexible lens of the human eye, embedded in the "capsular sac" and attached by laterally radiating zonula fibers to the ring-shaped ciliary muscle, loses flexibility and adaptivity for near or far vision, as we age. Presbyopia is the only refractive error that is considered to be progressive in nature.

Refractive surgery has been widely used in cornea reshaping and improving the eye's refractive power via high-power UV excimer laser (wavelength, $\lambda=193 \mathrm{~nm}$ ) that can break organic molecular bonds without damaging adjacent cells. Several different excimer laser refractive procedures have been devised. The most common of them are: photorefractive keratectomy (PRK) and most recently TransPRK, and laser assisted in situ keratomileusis (LASIK). Categorically, laser refractive surgery has enjoyed much popularity in performing LASIK and PRK on the cornea, with generally excellent results. However, there are some restrictions in performing it for myopia correction and, as an example; possible contraindications for LASIK are the cases of thin cornea or keratoconus disease. LASIK is a technique that involves an initial incision and flap formation to the cornea, either by blade (microkeratome) or by laser (femtosecond, fs-laser) and subsequent laser ablation for the prescribed corneal thickness removal. As it is well known, in cases where the level of myopic treatment is relatively high, the laser ablation depth is greater and therefore LASIK is advisable for corneas with thickness of at least $510 \mu \mathrm{m}$. The long experience in refractive surgery and the evaluation of the accumulated clinical outcomes generated substantial efforts to develop new methods in an attempt to enhance the therapeutic outcome. A major shift in myopia treating philosophy is slowly emerging, as the limitations of keratorefractive surgery become more evident [3].

The new trends in vision correction include the use of ocular implants as alternatives to treat high myopia, since they provide potentially better predictability and optical quality than cornealbased laser refractive surgery. For example, according to Katsanevaki et al., phakic intraocular contact lenses (ICLs), as well as multifocal and accommodating lenses are dramatically changing the way ophthalmologists perform refractive surgery [4]. Moreover, it is worth noting that as Mol et al. reported [5], special fabricated toric lenses of sphero-cylindrical shape can be implanted to correct high astigmatism levels. The last generation of toric intraocular lens delivers excellent rotational stability and a high level of spectacle independence for astigmatic cataract patients [6].

The interested reader can find several reviews on both research and clinical practice of laser-based myopia correction with a thorough literature search. Therefore, in this work we concentrate our effort to display basic and applied research efforts, as well as the ongoing needs and achievements for the laser-based correction of some other vision problems, in addition to refractive and diffractive errors. Furthermore, some remarks on myopia correction are sporadically presented, just for correlation with the reviewed novelties in laser applications in ophthalmology.

At this point, it is necessary to denote some non-refractive visual problems and their treatment modalities to possibly refine an alternative route for vision correction, based on laser action on intraocular implants. For a better understanding of new perspectives, based on biophotonic interventions in special visual disorders, we briefly present some basic notes on what cataract is and how the ophthalmologists face this problem.

\section{Cataract and IOLs: From conventional to novel prospects}

\section{What is cataract?}

It is well known that the eye's lens accounts for approximately onethird of its total refractive power. The cells of the human crystalline lens are normally arranged in a way that it is penetrable to visible light radiation, without causing significant phenomena of diffusion and scattering. To ensure transparency in the visible part of the electromagnetic spectrum, the human crystalline lens has no blood vessels and connective tissue. However, there are several pathologies that contribute to opacity of the lens. For instance, over time lens can become less transparent and may form a clouding, milky structure called cataract, preventing the clear light focusing on the retina and resulting in vision loss. The term "cataract" is derived from the Greek

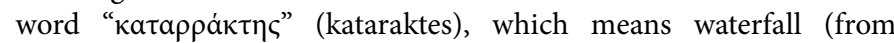
katarassein = to dash down, down-rushing) and from the later Latin word cataracta. According to the World Health Organization (WHO), cataract accounts for about half of all cases of blindness worldwide, while an estimated 20 million people suffering from bilateral cataracts $[7,8]$.

The cataractous lens effect on the visual quality of an image is artistically illustrated in Figure 1. Roughly, it seems that such illustrations reflect the patients' visual perception in a realistic way, although it is difficult to measure objectively their imaging clarity. An interesting work on this issue was published in 2006 by Michael Marmor as a special article [9]. Marmor, based on his medical knowledge and on computer simulations, tried to describe the effects of Claude Monet's cataract and Edgar Degas' retinal disease on their visual perceptions and the correlation between their diseases and painting styles [9].

Some cataracts are of idiopathic etiology. However, the first and foremost reason for cataract formation is age and thus cannot been prevented. Since lens' proteins are not restored during a person's life, and due to occurrence of oxidative processes, agglomerates are created. This effect produces turbidity, especially in the lens center, resulting in cataractous vision. Cataract can be classified into three types, based on the position in which appears. The significant hardening and yellowing of the lens, resulting in cloudiness in the central part, is known as a nuclear cataract. Nuclear cataracts are usually associated with aging and occur mainly in people of over 55 years old. Changes in the lens' cortex ionic composition lead to incomplete hydration of its fibers and

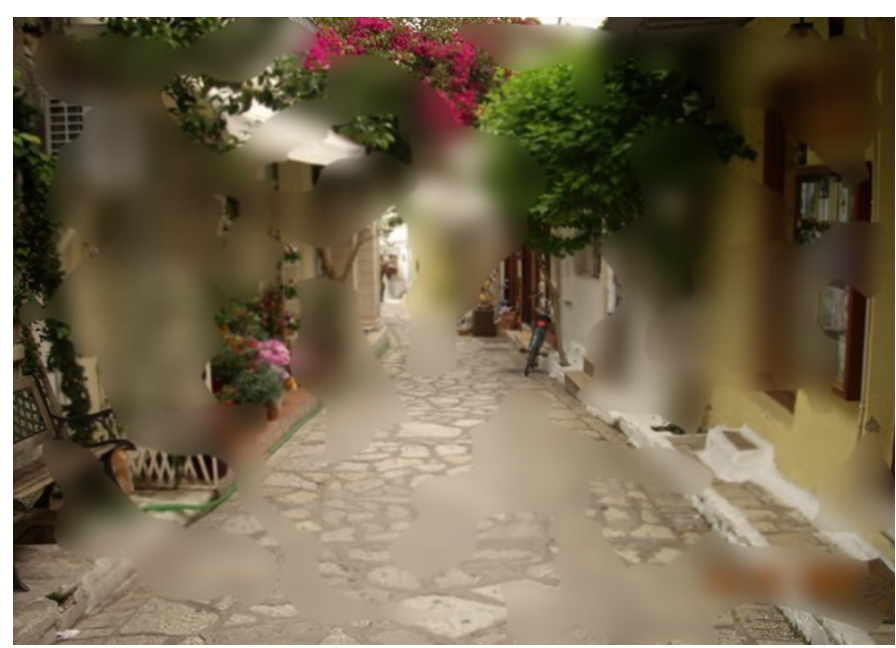

Figure 1. Representation of blurry vision in case of cataract 
cause cortical cataract. Finally, possible migration of epithelial cells into the area behind the capsule bag leads to posterior sub-capsular cataract $[10]$.

There is not any pharmaceutical or lens-based approach to treat cataract except surgery, where usually the cloudy natural lens is replaced by an artificial one (IOL). During the decades of cataract surgery and depending on the country of application, the intraocular implant insertion is not the only procedure. Briefly, some terms and their definition explain the cataract removal approaches:

(a) Aphakia, which means without lens. Older patients did not get implants, so they had to wear extreme hyperopic correction eyeglasses. This procedure is uncommon nowadays.

(b) Pseudophakia, which means with fake lens. In this case, an IOL implant replaces the natural lens, after cataract extraction.

(c) Phakic lens. In this case a lens implant is inserted, while leaving the crystalline lens intact.

\section{IOL types}

For many years, the removal of opaque human lens was being performed through an incision normally larger that the lens dimensions. Nowadays, phacoemulsification is a semi-invasive technique that breaks the cataractous lens by ultrasounds and afterwards the small fragments produced are suctioned through a small incision. A relatively flexible IOL is then inserted in the capsular bag by the same incision. According to Artal, cataract is an example of a disease in which a better understanding of the eye's optics leads to improved vision with better IOLs [11].

After the first successful IOL implant in a 45-year-old lady, by English ophthalmologist Sir Harold Ridley in 1949, IOLs have undergone continuous changes in their design and materials, defining several generations. Apart from the individual implant differences, an IOL has two main functional parts: the centrally arranged refractive area used for imaging and the outer parts, i.e. the haptics [12]. The haptics enable lens' fixation in the capsular bag. Various haptics designs have been developed to achieve strong stability (Figure 2), preventing lens' displacement inside the eye.

Several IOL types have been developed, where different light distributions on the pathway from the lens to the retina are prescribed. The lenses usually are characterized by their ability to provide good distant and near vision, to protect from harmful UV radiation, etc. Regarding the last issue, it has to be mentioned that as the natural crystalline lens absorbs strongly the radiation from $315 \mathrm{~nm}$ up to 400 nm (UVA), after cataract surgery and crystalline lens' removal, the retina is left exposed to these wavelengths. Therefore, the IOL to be inserted must be able to filter the UVA spectral area to protect retina. This is accomplished by adding UV absorbing chromophores during the polymerization of the IOL's material. a)

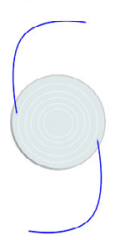

b)

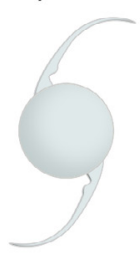

c)

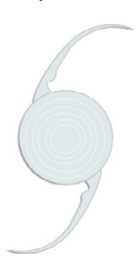

d)

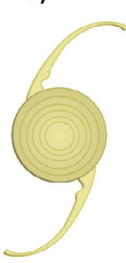

e)

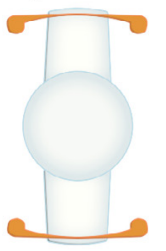

f)

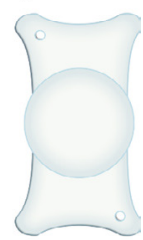

Figure 2. Representative forms of commercial intraocular lenses and their haptics. a), b) and c) IOLs are multifocal. d) is yellow due to its UV filter. e) is "accommodating" f) is aspheric
In order to satisfy focal demands of a wide range of distances (long distance, medium and short), multifocal IOLs have been developed. These are single lenses that have two different power values simultaneously $[3,13]$ and can be divided into two groups:

(a) Diffractive multifocals: which separate the incoming light into two different focal points, one for near objects and one for distance ones. Diffractive IOLs take advantage of diffraction caused by small, closely spaced, annular grooves cut into the lens surface that cause a large number of foci on the lens, while most of the power goes into the first two foci. By adjusting the spacing and shape of the grooves, the optical properties of the diffractive IOL can be manipulated.

(b) Refractive multifocals: which have a refracting surface with one or more focal points, usually through a series of concentric zones with different optical powers. Therefore, these IOLs present variations in zone size, number of zones and power distribution.

Recently, a group of medical doctors published an extensive overview of good clinical practice and general recommendations for multifocal IOLs available in the U.S., mentioning also available IOLs types outside the U.S., such as designs with trifocals or toric multifocal IOL combinations [14].

In modern IOLs' fabrication, besides diffractive-refractive multifocal lenses providing good distant and near vision, a new design has developed for intermediated vision, the so called apodization. An apodized pattern usually comprises 9 concentric steps of gradually decreasing step heights, from $1.3 \mu \mathrm{m}$ high centrally to $0.2 \mu \mathrm{m}$ peripherally [15]. These lenses are either made of PMMA, dyed with an UV blocking substance or of newer and more flexible materials, such as silicone and acrylic, which can be folded and placed through a smaller incision in the eye. Recently, the use of apodizing technology to design new refractive or diffractive multifocals improved the outcome but despite the progress made, the implanted lenses are not perfect yet.

Moreover, an interesting IOL type gives the possibility to correct refractive lens' power after it has been inserted in the eye, the socalled light-adjustable IOL. This three-piece implant is made of a unique silicone matrix, containing embedded photosensitive silicone macromeres. The lens is inserted through an incision using standard surgical techniques and the patient must return after the eye has healed. Then, the lens' power is adjusted to the patient's specific needs by polymerization of its silicone macromeres, using a low-intensity UV beam. Un-polymerized material diffuses into the treated areas, thickening the lens in specific, needed areas. The lens' material is designed to respond in a predictable manner according to the duration and intensity of light delivered, while lens' power adjustment typically lasts less than two minutes.

\section{Aspects to consider}

The choice of IOLs' materials includes biocompatible artificial polymeric materials, as polymethyl-methacrylate (PMMA), silicon, hydrophilic and hydrophobic polymers that ideally should never need to be replaced. However, the materials and methods for IOL fabrication have been correlated with some postoperative complications such as diffractive aberrations, capsular opacification or discoloration. Therefore, the main factors in the IOL material's choice include biocompatibility with the natural ocular tissues (including checks for cellular proliferation on the lens' surface, opacification of the posterior capsule, etc.), foldability, market demand and requirements, costs of the material and of the suitable production methods and ease of working with the material (brittleness or propensity to cracking or tearing). 
Furthermore, imperfections in wound healing and lens positioning may also create potential post-surgery refractive errors, such as farsightedness, nearsightedness, and astigmatism.

In addition to these side effects, visual accommodation is normally lost with a conventional IOL implant and the patients have to choose whether they want to have their distance vision corrected by the IOL and wear reading eyeglasses for near vision (which usually happens) or to select a power that would permit them to be near-sighted through the IOL and have a distance correction in their eyeglasses. Sometimes, a few patients also choose a monovision strategy in which one eye is implanted with an IOL for distance vision and the other with an IOL power that can provide some near vision without eyeglasses correction [16-18]. Certainly, achieving eyeglasses independence is challenging and, for certain patients, this is reflected in choosing multifocal IOLs. Patients who are not good candidates for multifocal IOLs may be better suited to one of the newer generations of aspheric monofocal IOLs, which can offer improved functional vision and better contrast sensitivity postoperatively. However, it should be stated that not every currently available IOL is suitable for every patient due to the complexity of lifestyle choices and personality dynamics or the inherent eye anatomy and physiology [14]. Furthermore, do not forget that incorrect lens power remains one of the top most common reasons for foldable IOLs explantation.

Another critical point in the design of novel IOLs is the minimization of the ocular aberrations. It is known that the average sphericity of the normal human cornea is positive and remains stable throughout life, while the lens' spherical aberration (SA) changes with age [19]. Normally, the crystalline lens of young eyes has an overall negative SA that compensates for the positive cornea's SA. A quality image and proper vision is produced as light rays are focused on the retina. On the contrary, in older eyes the crystalline lens loses its ability to compensate for corneal SA, total ocular SA becomes increasingly positive, and the resulting aberrations cause blurred vision and reduced contrast sensitivity [20]. SA is associated with glare, halos and decreases the contrast sensitivity, especially in scotopic conditions. Consequently, even people with good Snellen visual acuity could have decreased life quality and driving safety, while the onset of cataract worsens the related problems, especially the Achilles' heel of emmetropia, the functional intermediate visual acuity.

\section{From conventional to novel use of IOLs}

Prosthetic lens insertion has been developed as a conventional practice nowadays. Additionally, having a plethora of IOL choices, modern attempts propose their implantation in addition and close to the human lens to treat high levels myopia or hyperopia; if refractive surgery (e.g. LASIK) is not possible (e.g. very thin corneas and extreme abnormality in shape). Furthermore, several factors such as prolonged healing times, corneal irregular astigmatism, halos at night and the expensive and maintenance-dependent practice of the worldwide used ArF excimer laser, have promoted the introduction of IOLs for refractive surgery purposes. According to Lane et al. [3], it is likely that in the near future refractive surgery will encompass an increase in anterior chamber IOLs' use, offering several advantages for the correction of ametropia and presbyopia, while LASIK and PRK will remain important procedures for the correction of low ametropia and for refining pseudophakic IOL results, such as astigmatism.

It seems that soon, instead of applying "refractive surgery on cornea", the concept of "refractive surgery on intraocular implant" will be feasible, while leaving cornea untouchable. For example, in a novel intraocular refractive surgery, the so called PIOL (Phakic IntraOcular Lens) is proposed to be implanted behind the cornea and in front of the natural crystalline lens to improve vision. PIOL is implanted into the eye without taking out the natural lens and is effective in replacing high eyeglasses prescriptions, while is also widely used to treat younger patients who are not suitable for laser eye surgery [21]. In a recent study [22], Kamiya et al. evaluated retrospectively the visual and refractive outcomes of one type of PIOL, namely a posterior chamber PIOL with a central hole (hole ICL), implanted in eyes with relatively low anterior chamber depth (ACD). They concluded in their multicenter case series that ICL implantation was good for the correction of myopia and myopic astigmatism, in all outcomes of safety, efficacy, predictability and stability. The Japanese group [23] applied the small incision lenticule extraction (SMILE) technique for the correction of myopia and myopic astigmatism in patients, using a $500 \mathrm{kHz}$ fs-laser system and reported that SMILE was also beneficial throughout the 1-year observation period.

The reader should have in mind that even the best lens possible is still not the perfect one; perfect lenses could only be the customized ones. The production of fully customized IOLs for each patient is innovative and remarkably interesting, both on a scientific level and on a commercial basis. As a consequence of the extended use of intraocular implants, there is a great demand for customized IOLs, specially made for everyone just before the cataract operation. However, the customized IOL's production is complicated, expensive and time-consuming. Recall for instance, that multifocal IOLs are produced mostly by diamond turning, while this shaping technique has certain drawbacks, like long machining times and thermal stress in the material. Therefore, a hypothesis was addressed a couple of years ago. What if IOL-polymers could be manipulated by "cold" laser ablation and, therefore, a novel manufacturing process based on this approach be implemented $[15,24,25]$ ? Certainly, such a process would have to provide a good quality, e.g. low surface roughness by reducing both the surface and bulk polymer damages but it would also require high efficiency, enough to allow economical IOLs' production.

\section{Laser - IOLs interaction for polymer surface modification}

The basic, novel scheme in IOL's structuring process is based on soft polymer laser ablation for patterning or surface modification, to create well prescribed micron-sized apodized patterns. This could provide an alternative route for IOL's material cutting and etching, instead of using the techniques of injection molding and lathe-cut, which have been correlated with some postoperative complications such as posterior capsular opacification [26,27]. The idea is to offer ophthalmologists a tool for customized laser-IOL patterning according to patient needs, including wavefront, multifocal and astigmatic corrections, whether the lens is inside the eye (e.g. after a cataract surgery) or outside of the eye.

In the polymeric materials' processing literature, a number of groups have studied the sophisticated aspects of laser action for polymers' surface and/or bulk structuring and modification [28,29]. Our group used its experience that accumulated through decades of micromachining research of synthetic polymers in various fields of everyday life, as well as in sophisticated technological fields (e.g. in optoelectronics, photolithography and optical data storage devices) and in several biomedical applications (biocompatible polymers as contact/ IOLs, dental implants and bone prostheses), and for the last decade has been studying the laser ablation technique for IOL's surface structuring $[15,28,30-32]$. 
Among the various laser sources, excimer lasers have been widely used in polymeric material applications, such as e.g. etching, perforation, direct printing, micro-processing and thin film deposition [26]. Our first efforts for customized IOLs included the use of ArF excimer laser, since it is still the dominant source for cornea ablating in refractive surgery, while it is also considered appropriate for reshaping polymers in other technological applications. However, the analysis of our previous results showed that the UV excimer laser radiation $\left(\lambda=193 \mathrm{~nm}, \mathrm{t}_{\text {pulse }}=8 \mathrm{~ns}\right.$, photon energy $\left.=6.42 \mathrm{eV}\right)$, at relatively low energy fluencies (representing conditions similar to those used in refractive surgery), can create conical microstructures on both PMMA and acrylic IOL ablated surfaces [32,33]. Figure 3 presents the same phenomenon in some of our newer experiments. The formation of the conical microstructures (spikes) could be the result of the so-called shielding effect. According to it, material's microstructures [34] or impurities [35] are not ablated under the wavelength and/or the energy fluence used. Hence, they "protect" the underlying layers, resulting in spikes' formation. Carbon microparticle aggregates can also lead to the same result, as according to Silvain et al., their high thermal conductivity leads to faster thermal ablation of the surrounding material [36]. Furthermore, our analysis reveals wavy patterns in the crater's floor (Figure 3(b)), with the distance between two consecutive crests (fringes) being approximately $64 \mu \mathrm{m}$, a value significantly higher than the nanometer wavelength scale of the beam. As a result, these patterns cannot be attributed to wave interference phenomena of the incident and scattered rays. One possible explanation for this phenomenon is that as the polymer is getting colder, the mechanical waves generated by temperature gradients are solidified. Another is that since the floor of the crater is exposed to the beam's wavefront, and as a result to higher intensity due to the Gaussian profile, it reaches higher temperature than its walls. Hence, the floor tends to dilate more than the surrounding material and creates ripples which solidify during the cooling process. Finally, the well-defined and relatively clear crater's walls have to be mentioned.

Furthermore, several solid-state laser sources have been used, covering from UV to mid-IR wavelengths, as potential alternatives to the ArF excimer laser that has already been used in myopia correction $[15,28,31]$. A key parameter for characterizing the laser-polymer interaction is the ablation threshold, defined as the minimum laser energy per unit surface (radiant exposure - $\mathrm{H}$ ) required to provoke detectable changes in the material. A low ablation threshold is generally favorable for minimizing collateral damage near the ablation area. However, even though laser-based micro-relief aspects could be well controlled, biomedical applications demand more studies oriented on light refraction/diffraction and polymeric materials biocompatibility. Moreover, the laser ablation behavior depends on the IOL micro- (a)

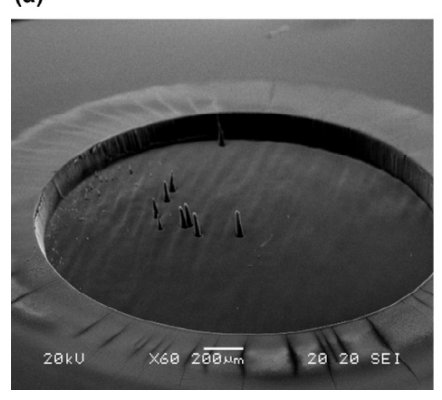

(b)

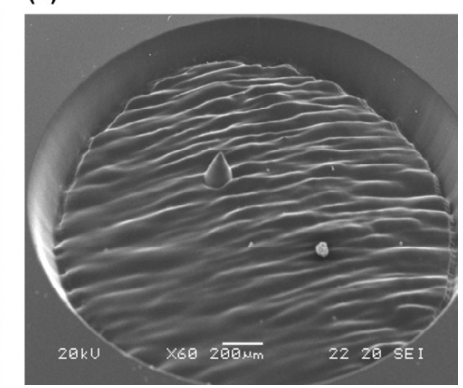

Figure 3. SEM images of irradiated acrylic IOLs with $\lambda=193 \mathrm{~nm}$, frequency $(\mathrm{f})=5 \mathrm{~Hz}, \mathrm{H}$ $=0.2 \mathrm{~J} / \mathrm{cm}^{2}$ : (a) (Alcon MA30BA, $\left.22.5 \mathrm{D}\right) \mathrm{N}=1500$ pulses and (b) (Storz H60M, $\left.15 \mathrm{D}\right) \mathrm{N}$ $=1500$ pulses. The conical microstructures are visible in both cases environment e.g. whether the implant' material is outside or inside the eye.

As far as this problem is concerned, a promising solution could be the non-invasive modification of an implanted IOL by using the appropriate laser light to modify in situ its optical parameters, while preserving the optical quality. Moreover, the in situ IOL manipulation can modify IOL's diopters in order to account for surgical errors, tilt, decentration or a change in the physical characteristics of the eye. Without doubt, before any experimental effort for in situ IOL ablating, the qualitative aspects of laser-polymer interaction must be examined carefully [15].

The first step is the selection of the appropriate laser wavelength. It has to be based on the correlation of the human eye's optical properties and the transmission of the optical spectrum on various ocular media, from the cornea to retina. For non-invasive, in situ optical performance modification of an implanted IOL, the appropriate wavelength seems to be in the range of 315 to $390 \mathrm{~nm}$ (near-UV or UVA). Nevertheless, the use of UV light photons must be considered with skepticism, as it is well known that the radiation starting at $200 \mathrm{~nm}$ and up to $400 \mathrm{~nm}$ is harmful to the retina. Fortunately, the light penetration through ocular media varies depending on the wavelength and the type of tissue as following: UVC radiation $(200-290 \mathrm{~nm})$ is absorbed by the cornea, UVB radiation $(290-320 \mathrm{~nm})$ is absorbed by the lens capsule, and UVA radiation $(320-400 \mathrm{~nm})$ is absorbed by the lens' stroma [37]. Hence, the near-UV or UVA photons reaching up to the human eye's crystalline lens do not penetrate deeper, and thus the retina is protected. Note that after cataract surgery a significant part of the natural UV filter function is lost. For this reason, a UV-blocking agent is incorporated routinely while manufacturing the IOLs. For example, IOLs made by traditional polymeric materials such as PMMA are suitably stained with substances e.g. yellow azo-dyes which block UV and blue-light radiation [38]. This fact complicates furthermore the research efforts for UV laser photons use to modify the implanted IOLs.

The UV filtering on human lens level, either by natural crystalline lens or by suitably dyed IOLs, is particularly important. Recently, Spyratou et al. [39] investigated the possible effect of ionizing radiation exposure for patients and for occupationally exposed medical staff in interventional radiology and cardiology procedures, by comparing the transmission spectra of yellow azo-dyed acrylic IOLs in pre- and post-irradiation conditions. They reported that the X-ray irradiation at clinical doses used in interventional radiology and cardiology procedures can significantly affect the IOLs' filter protection against the harmful UV and short wavelength light, while the transmittance in the visible region is slightly reduced.

The Nd:YAG laser is also promising for IOL surface structuring. Our previous, in air experiments studied craters' morphology, to investigate the use of UV laser radiation for customized IOLs' apodization or for in situ ablation. We performed laser ablation experiments on commercially available hydrophobic acrylic IOLs (e.g. Alcon MA30AC, MA30BA and MA60BM models) and yellow azo-dye IOLs (Alcon SN60WF and SN60AT). The laser source was the $3^{\text {rd }}$ harmonic of a solid state, $\mathrm{Nd}$ :YAG laser, providing laser pulses at $\lambda=355 \mathrm{~nm}$, with pulse duration of $10 \mathrm{~ns}$ and photon energy of $\sim 3.49 \mathrm{eV}$, at low and moderate laser radiant exposure levels. The morphology of the ablated IOLs' surface was examined using a conventional optical microscope (Leitz SM-LUX, Hicksville, NY, US) and a scanning electron microscope (SEM, Fei Innova Nanoscope). 
Figure 4(a) is indicative of the correlation between the pulses' number and crater's diameter. As expected, the more the pulses are, the deeper the floor of the crater lays. Hence, the scattered rays cannot escape and ablate further the crater's walls, increasing its diameter. Moreover, as shown in Figure 4(b), the surface of the walls appears perforated by pores with a spongy texture. The ablated surface shows relatively amorphous, while molten residues have been dropped around the crater's boundaries. Although the exact synthesis of the IOLs is not easily available due to patent and confidentiality reasons, their chemical structure includes $\mathrm{C}-\mathrm{O}, \mathrm{C}=\mathrm{O}$ and $\mathrm{C}-\mathrm{H}$ bonds. As it is known, the intramolecular chemical bond of $\mathrm{C}-\mathrm{O}$ and $\mathrm{C}=\mathrm{O}$ require 3.71 and $8.28 \mathrm{eV}$ to be broken, respectively; while the $\mathrm{C}-\mathrm{H}$ bond requires $4.28 \mathrm{eV}$ [40]. As a result, photons with lower energy can break the former bonds only by multiphoton processes, observed mainly in shorter pulses. In cases of higher energy values than the needed ones for intra- and intermolecular bond breaking, the excess is converted into thermal / kinetic energy of the debris detached from the crater. The recoil momentum of these debris (Figure 5(a)) manifests itself in the form of mechanical waves, which generate cracks around the crater as they propagate, producing the characteristic asteroid shape of Figures 4(a) and 5. It should be also taken into account, that sharp discontinuities (and thus cracks) on polymeric materials lead to local increase in the mechanical stresses produced. These, along with the decrease of intermolecular forces between the macromolecules on each side of the clefts and the mechanical waves at the floor of the crater lead to further cracks' opening. Since similar patterns were also observed in other hydrophobic IOLs, the possibility to have been caused due to a single IOL's material failure is minimized.

Regardless of a good surface quality achieved, undesirable side effects could possibly be provoked by the ablation debris. Therefore, the transport of reliable research data to clinical applications meets an obstacle. What about ablation debris inside the eye? Remember that, by definition, laser ablation is the process of removing material from a solid (or occasionally liquid) surface by irradiating it with a laser beam (Figure 5). Depending on the incident laser energy fluence and the absorptivity of the target, the IOL is locally heated by the absorbed laser energy and evaporates or sublimates or is converted to plasma that rapidly expands in the form of shock waves. Obviously, any unwanted alteration or material debris deposition induced by the incident laser radiation on polymer surface is an undesirable effect, as a potential source of light scattering and/or a cause for cell attachment and clouding of implanted IOLs.

Another problem that has to be faced for the in situ laser ablation of IOL polymers is the superposition of the consecutive Gaussian laser pulse spots on the surface, with potentially important consequences in the effective ablation pattern. Some years ago, preliminary experiments were performed in our lab in an effort to create circular zones on the lens polymer surface, close to apodization techniques used by IOL manufacturers. We performed laser ablation on commercially available hydrophobic and hydrophilic acrylic IOLs with the $5^{\text {th }}$ harmonic of a Q-switched Nd:YAG laser $(\lambda=213 \mathrm{~nm})$ [28]. Prompted by them, we mention two polymer surface patterning problems that have to be taken in consideration: i) as the laser incidence is always perpendicular to the sample, the ablation patterns on spherical surfaces are altered by variations in ablation efficiency due to changes in the beam's incidence angle, as a function of the distance from the lens' center, ii) overlapping of adjacent focus spot locations, corresponding to consecutive laser pulses during the ablation ring raster scanned, shows a focus spot overlap of approximately $33 \%$ in some cases (Figure 6) [41]. (a)

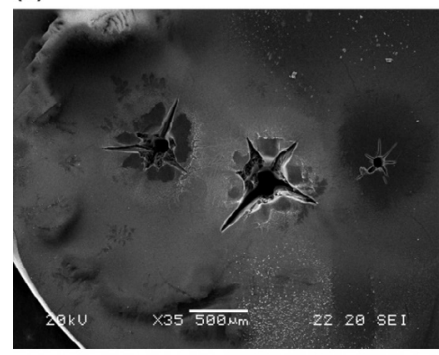

(c)

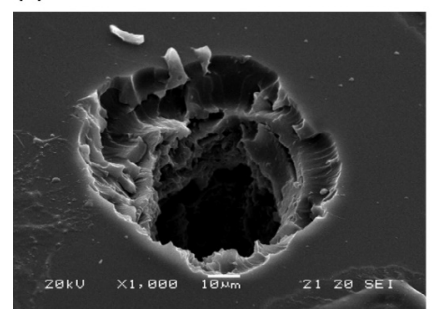

(b)

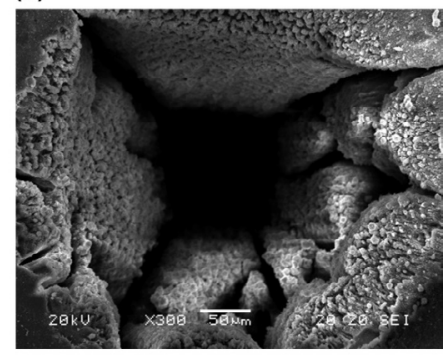

(d)

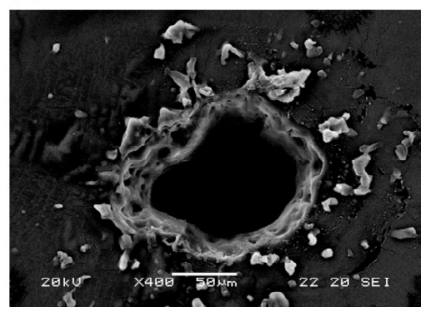

Figure 4. SEM image of irradiated acrylic IOLs with $\lambda=355 \mathrm{~nm}, \mathrm{f}=10 \mathrm{~Hz}$ : (a) (Alcon MA30BA, $30 \mathrm{D}) \mathrm{F}=5.3 \mathrm{~J} / \mathrm{cm}^{2}, \mathrm{~N}=1200,3000$ and 600 pulses, from left to right, respectively, (b) (Alcon SN60WF, $18 \mathrm{D}$ ) $\mathrm{F}=2.7 \mathrm{~J} / \mathrm{cm}^{2}, \mathrm{~N}=3000$, (c) (Storz H60M, $17 \mathrm{D}$ ) $\mathrm{F}=2.7 \mathrm{~J} / \mathrm{cm}^{2}, \mathrm{~N}=300$ and (d) (Alcon SN60WF, $10 \mathrm{D}$ ) $\mathrm{F}=2.7 \mathrm{~J} / \mathrm{cm}^{2}, \mathrm{~N}=1200$, after UVA irradiation $\left(\lambda=370 \mathrm{~nm}, \mathrm{~F}=90 \mathrm{~mJ} / \mathrm{cm}^{2}\right)$

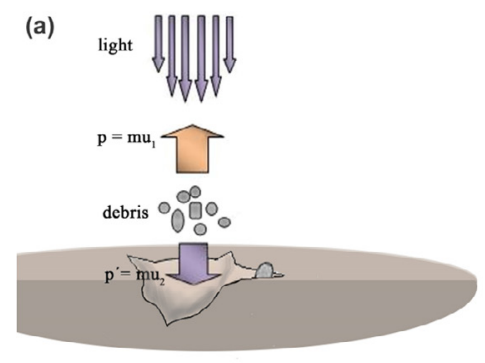

(b)

Figure 5. (a) Schematic representation of the creation of star-shaped craters and (b) SEM image of an irradiated acrylic IOL (Alcon MA30BA, $30 \mathrm{D}$ ) with $\lambda=355 \mathrm{~nm}, \mathrm{f}=10 \mathrm{~Hz}, \mathrm{~F}$ $=5.3 \mathrm{~J} / \mathrm{cm}^{2}$ and $\mathrm{N}=600$ pulses

(a)

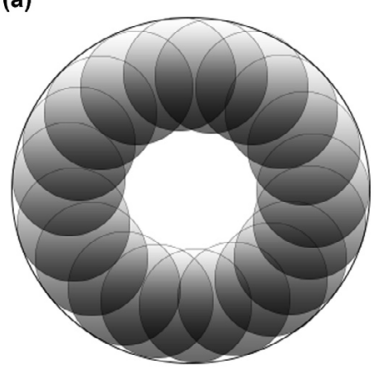

(b)

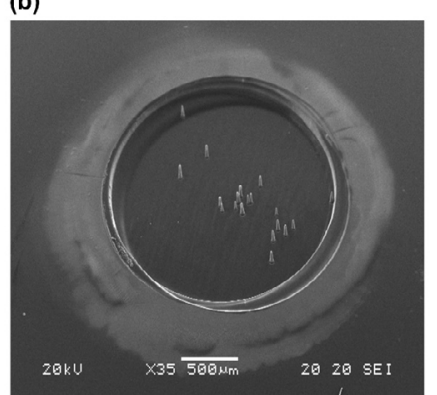

Figure 6. (a) Artistic illustration of pulses irradiating repetitive / scanning the periphery of an IOL. (b) SEM image of an IOL that was accidentally moved during its irradiation (Alcon SN60WF, $18 \mathrm{D}, \lambda=193 \mathrm{~nm}, \mathrm{f}=50 \mathrm{~Hz}, \mathrm{~F}=0.2 \mathrm{~J} / \mathrm{cm}^{2}, \mathrm{~N}=1500$ pulses). The pulses' overlapping is noticeable, especially at the bottom-right of the crater

Finally, we must emphasize the laser safety problems raised using the ocular media penetrating light at $\lambda=355 \mathrm{~nm}$. The interested reader could refer to the International Commission on Non-Ionizing Radiation Protection (ICNIRP) 2004 guidelines ("Guidelines on limits of exposure to ultraviolet radiation of wavelengths between $180 \mathrm{~nm}$ and $400 \mathrm{~nm}$ (incoherent optical radiation)," 2004) that give the safety limits 
for energy fluence of $355 \mathrm{~nm}$ radiation based on the potential hazard for photochemical cornea damage.

\section{Laser - IOLs interaction for refractive index modification: A brief overview}

In the past decade, as new ideas came to the scene, our research group progressed to a new, non-ablative approach to modify IOL optical behavior/power after the lens has been inserted into the eye, based mainly in efficient and safe alteration of implanted polymer's refractive index (RI). Our first results indicate a small RI modification of polymeric ocular lenses and PMMA plates, resulting in a small refractive power change $(\sim 0.25 \mathrm{D})$ [42]. We hypothesize that the laser interaction with IOLs depends on both optical and physico-chemical material's properties, while the exact mechanism of photo-induced modification of refractivity has to be further elucidated before any attempt to translate this research into in vivo and, furthermore, to clinical applications.

A brief overview of the non-invasive IOL RI modification era follows. Träger et al. in 2006, presented the idea of creating novel polymers, suitable for IOL's fabrication, whose RI can be changed noninvasively by photo-induced processes [43]. According to them, RI changes are achieved by cross-linking or cleaving chemical bonds in a $[2+2]$ cycloaddition or cycloreversion, respectively. Recently, different theories regarding fs-laser and material interactions that affect the RI have been reported. It is important to notice that, in ultra-short pulsed laser processing, absorption has no significant role and, therefore, the relevant laser-polymer interaction mechanisms are more complicated. For example, several scientific reports on material processing and polymer micro-structuring by short-pulsed laser ablation, reveal that laser pulses are linearly absorbed in polymers through electronic transitions, while ultrashort picosecond [12] and femtosecond laser pulses are delivered to the sample via nonlinear multiphoton absorption $[44,45]$. Sahler et al. in their research reported that the IOL refractive properties can be customized after implantation, using a fslaser, based on the beam's parameters and the interaction mechanisms along with the IOL material's optical properties [46]. According to them, fs-laser alters the hydrophilicity of defined zones within an IOL; and since hydrophilicity change leads to large, repeatable and homogeneous change in refractive characteristics, an RI shaping lens can be built within that zone. This change in hydrophilicity occurs when the polymeric material is immersed in an aqueous medium and is exposed to fs-laser radiation. Moreover, prof. Wayne Knox, working in the field of ultrafast lasers for more than 40 years, along with the team of scientists at the University of Rochester proposed a new procedure for vision correction, which will be safer and more flexible than LASIK [47]. This new technique is based on using a laser to induce RI change, named by the acronym LIRIC (Laser-Induced Refractive Index Change). The LIRIC procedure changes the focusing capability of the cornea's internal optics noninvasively [48]. The Knox group suggested that the laser-induced crosslinking within a hydrophilic material creates an RI increase [49].

Figure 7 gives a schematic representation for the RI modification of an IOL by using a focused fs-laser at $\lambda \approx 800 \mathrm{~nm}$ that is transmitted through cornea and lens to retina. For safety reasons, the defocused laser beam is calculated to further hit retina in lower than the maximum permissible exposure levels (MPE).

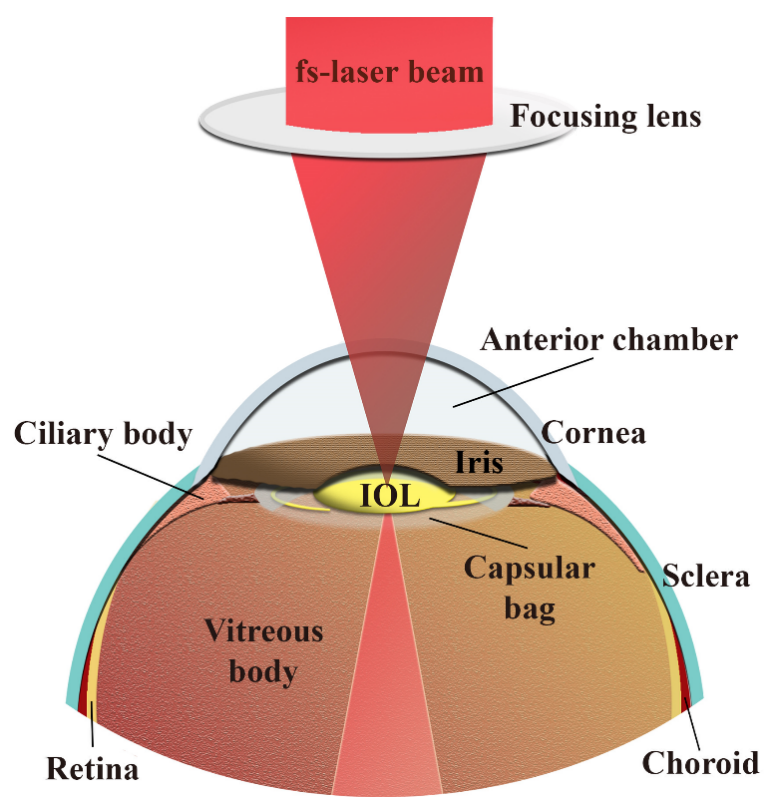

Figure 7. Schematic representation of the modification of the refractive index of an implanted intraocular lens by a femtosecond laser

\section{Concluding remarks and perspectives}

As already mentioned, currently, the only "treatment" for the cataract vision disorder is surgery. In the new cataract era, several advances involve improvements in surgical techniques, IOL technology and preoperative evaluation. Summarizing the innovative expectations in vision correction, new materials that are expandable, lightadjustable, thermodynamic and photochromic should increase the use of IOLs within the next few years, while laser ablation for IOLs surface modification also seems a very promising procedure. However, before any attempt to analyze the possibility of using laser ablation for IOL surface modification, it is reasonable to recall the basic experimental and theoretical knowledge addressed to laser ablation for polymer surface microstrucruring. The laser fluence, the repetition rate and the number of pulses must be determined carefully, taking into consideration the material's properties for optimum etch characteristics. The IOL surface patterning in the level of $0.2 \mu \mathrm{m}$ (nanometer scale!) requires exceptional precision. We wonder if this precision is feasible. The quality of laser ablation is supposed to allow satisfactory penetrability in the visible light and to prevent undesirable results afterwards the IOL implantation in the patient.

Apart from laser interventions in IOL optical power customization, the emerging perspective of "IOLs based refractive surgery" is also very promising. This procedure concerns the improvement of the IOL optical properties by focusing the appropriate laser beam for in situ polymer surface modification. If the laser light is not transmitted to the intraocular implant level, as for example for a range of UV wavelengths, the use of a conduit (e.g. optical fiber) is required to transport the beam directly to the intraocular contact lens or PIOL site, to allow sufficient power delivery and to achieve controlled ablation with minimal collateral damage to surrounding tissues.

Finally, femtosecond laser use has great potentials, when of course operated at emission levels that comply with the cornea's and retina's safety limits and biocompatibility is maintained. Its ability to create customized multifocal IOLs for near, middle and far eyeglasses-free 
vision, addressing each patient's specific needs, is innovative and worth exploring. Hence, the cost affordable manipulation of an IOL's diopters, through its refractive index change, is the feasible goal for a fs-laser with the appropriate intensity and wavelength.

\section{Acknowledgements}

The author G.K. would like to state that his part of this research has been co-financed by Greece and the European Union (European Social Fund- ESF) through the Operational Program «Human Resources Development, Education and Lifelong Learning» in the context of the project "Strengthening Human Resources Research Potential via Doctorate Research" (MIS-5000432), implemented by the State Scholarships Foundation (IKY) [Scholarship number: 2018-050-050214578]. The authors E.D, M.M. and A.A.S. gratefully acknowledge the funding by the "ELI-LASERLAB Europe Synergy, HiPER \& IPERIONCH.gr (HELLAS-CH)" MIS 5002735 (Co-financed by Greece and the European Union- European Regional Development Fund). The funding sources had no involvement in the study design; in the collection, analysis and interpretation of data; in the writing of the report; and in the decision to submit the article for publication.

\section{References}

1. Palanker D V, Blumenkranz MS, Marmor MF (2011) Fifty years of ophthalmic laser therapy. Arch Ophthalmol 129: 1613-1619. [Crossref]

2. Kohnen T, Strenger A, Klaproth OK (2008) Basic knowledge of refractive surgery: correction of refractive errors using modern surgical procedures. Dtsch Arztebl Int 105: 162-163. [Crossref]

3. Lane SS, Morris M, Nordan L, Packer M, Tarantino N, Wallace 3rd RB (2006) Multifocal intraocular lenses. Ophthalmol Clin North Am 19: 89-105.

4. Katsanevaki VJ, Tuft SJ (2007) Refractive surgery: what patients need to know. Br Med Bull 83: 325-339. [Crossref]

5. Mol IE, Van Dooren BT (2016) Toric intraocular lenses for correction of astigmatism in keratoconus and after corneal surgery. Clin Ophthalmol 10: 1153-1159. [Crossref]

6. Gyongyossy B, Jirak P, Schonherr U (2016) Rotational stability and patient satisfaction after implantation of a new toric IOL. Eur J Ophthalmol 26: 321-327. [Crossref]

7. Khairallah M, Kahloun R, Bourne R, Limburg H, Flaxman SR, et al. (2015) Number of People Blind or Visually Impaired by Cataract Worldwide and in World Regions, 1990 to 2010. Invest Ophthalmol Vis Sci 56: 6762-6769. [Crossref]

8. Organization WH. Global initiative for the elimination of avoidable blindness: action plan 2006-2011 2007:vi, 89 p.

9. Marmor MF (2006) Ophthalmology and art: simulation of Monet's cataracts and Degas' retinal disease. Arch Ophthalmol 124: 1764-1769. [Crossref]

10. Raj SM, Vasavada AR, Johar SRK, Vasavada VA, Vasavada VA (2007) Post-operative capsular opacification: a review. Int J Biomed Sci 3: 237-250. [Crossref]

11. Artal P (2014) Optics of the eye and its impact in vision: a tutorial. Adv Opt Photonics 6: $340-367$.

12. Heberle J, Klämpfl F, Alexeev I, Schmidt M (2014) Ultrashort pulse laser cutting of intraocular lens polymers. J Laser Micro Nanoeng 9: 103-107.

13. Optics of Intraocular Lenses n.d. https://www.healio.com/ophthalmology/refractivesurgery/news/online/\%7B6917d3d0-9c68-40b2-9494-fa0969104f3d\%7D/optics-ofintraocular-lenses (accessed December 15, 2019).

14. Braga-Mele R, Chang D, Dewey S, Foster G, Henderson BA, et al. (2014) Multifocal intraocular lenses: relative indications and contraindications for implantation. $J$ Cataract Refract Surg 40: 313-322. [Crossref]

15. Apostolopoulos A, Lagiou DP, Evangelatos C, Spyratou E, Bacharis C, et al. (2013) UV solid state laser ablation of intraocular lenses. Med Laser Appl Laser-Tissue Interact.

16. Davison JA, Simpson MJ (2006) History and development of the apodized diffractive intraocular lens. J Cataract Refract Surg 32: 849-858. [Crossref]

17. Glasser A, Campbell MC (1998) Presbyopia and the optical changes in the human crystalline lens with age. Vision Res 38: 209-229.
18. Jain S, Arora I, Azar DT (1996) Success of monovision in presbyopes: Review of the literature and potential applications to refractive surgery. Surv Ophthalmol 40: 491499. [Crossref]

19. Khanna A, Dhiman R, Khanna R, Rathore YS, Arun S (2015) Role of asphericity in choice of IOLs for cataract surgery. Delhi J Ophthalmol.

20. Guirao A, Redondo M, Artal P (2000) Optical aberrations of the human cornea as a function of age. J Opt Soc Am A Opt Image Sci Vis 17: 1697-1702.

21. Phakic Intraocular Lens Implantation Patient Information. R Coll Ophthalmol n.d. https://www.rcophth.ac.uk/wp-content/uploads/2015/02/Phakic-Intraocular-LensImplantation-Patient-Leaflet-April2017.pdf (accessed October 26, 2019).

22. Kamiya K, Shimizu K, Igarashi A, Kitazawa Y, Kojima T, et al. (2018) Posterior chamber phakic intraocular lens implantation in eyes with an anterior chamber depth of less than 3 mm: A multicenter study. Sci Rep 8: 13322. [Crossref]

23. Kamiya K, Shimizu K, Igarashi A, Kobashi H (2015) Visual and refractive outcomes of small incision lenticule extraction for the correction of myopia: 1-year follow-up. BMJ Open 5: e008268. [Crossref]

24. Sankar V, Kumar ST, Rao KP (2004) Preparation, characterisation and fabrication of intraocular lens from photo initiated polymerised poly (methyl methacrylate). Trends Biomater Artif Organs 17: 24-30.

25. Sen HN, Sarikkola AU, Uusitalo RJ, Laatikainen L (2004) Quality of vision after AMO Array multifocal intraocular lens implantation. J Cataract Refract Surg 30: 2483-2493. [Crossref]

26. Dyer PE (2003) Excimer laser polymer ablation: twenty years on. Appl Phys A Mater Sci Process 77: 167-173.

27. Wilson SE, Brubaker RF (1987) Neodymium: YAG laser damage threshold. A comparison of injection-molded and lathe-cut polymethylmethacrylate intraocular lenses. Ophthalmology 94: 7-11. [Crossref]

28. Lagiou DP, Evangelatos C, Apostolopoulos A, Spyratou E, Bacharis C, et al. (2013) UV laser ablation patterns in intraocular lenses. Seventeenth Int Sch Quantum Electron Laser Phys Appl.

29. Lasagni A, Cornejo M, Lasagni F, Muecklich F (2008) Laser ablation modeling of periodic pattern formation on polymer substrates. Adv Eng Mater 10: 488-493.

30. Serafetinides AA, Makropoulou M, Fabrikesi E, Spyratou E, Bacharis C, et al. (2008) Ultrashort laser ablation of PMMA and intraocular lenses. Appl Phys A 93: 111-116.

31. Serafetinides AA, Makropoulou M, Spyratou E, Bacharis C (2007) Alternatives to excimer laser refractive surgery: UV and mid-infrared laser ablation of intraocular lenses and porcine cornea. 14th Int Sch Quantum Electron Laser Phys Appl 6604: 13.

32. Spyratou E, Makropoulou M, Tsoutsi D, Zoulinakis G, Bacharis C, et al. (2012) Conical structures on acrylic intraocular lens (IOLs) materials after 193-nm excimer laser ablation. Mater Sci Appl 03: 414-424.

33. Serafetinides AA, Spyratou E, Makropoulou M (2011) Laser ablation and high precision patterning of biomaterials and intraocular lenses. 16th Int Sch Quantum Electron Laser Phys Appl 7747: 14.

34. Tokarev VN, Lopez J, Lazare S, Weisbuch F (2003) High-aspect-ratio microdrilling of polymers with UV laser ablation: experiment with analytical model. Appl Phys A 76: 385-396.

35. Hopp B, Bor Z, Homolya E, Mihalik E (1997) Investigation of conical structures created by ArF excimer laser irradiation of polycarbonate. Appl Surf Sci 109-110: 232235.

36. Silvain JF, Niino H, Ono S, Nakaoka S, Yabe A (1999) Surface modification of elastomer/carbon composite by $\mathrm{Nd}+$ :YAG laser and $\mathrm{KrF}$ excimer laser ablation. Appl Surf Sci 141: 25-34.

37. Walsh K (2009) UV radiation and the eye. Optician 237: 26-33.

38. Downes SM (2016) Ultraviolet or blue-filtering intraocular lenses: what is the evidence? Eye 30: 215-221.

39. Spyratou E, Antonakos J, Platoni K, Bacharis C, Makropoulou M, et al. (2017) Investigating the effect of ionizing radiation on intraocular lenses at clinical doses. $J$ Med Phys Appl Sci.

40. Bond Energies - Chemistry LibreTexts n.d. https://chem.libretexts.org/Bookshelves/ Physical_and_Theoretical_Chemistry_Textbook_Maps/Supplemental_Modules (Physical_and_Theoretical_Chemistry)/Chemical_Bonding/Fundamentals_of Chemical_Bonding/Bond_Energies (accessed February 11, 2020). 
41. Homolka P, Biowski R, Kaminski S, Barisani T, Husinsky W, et al. (1999) Laser shaping of corneal transplants in vitro: area ablation with small overlapping laser spots produced by a pulsed scanning laser beam using an optimizing ablation algorithm. Phys Med Biol 44: 1169-1180. [Crossref]

42. Bacharis C, Tsilikas G, Sianoudis I, Makropoulou M, Zoulinakis G, et al. (2018) Laserinduced refractive index modification of intraocular lenses. E-Journal Sci Technol 13: $89-96$.

43. Träger J, Kim HC, Hampp N (2006) Polymers for refractive index change in intraocular lenses: a novel approach for photo-induced tuning of focal length. In: Manns F, Söderberg PG, Ho A, editors. Ophthalmic Technol. 6138: 311-319.

44. Eaton SM, De Marco C, Martinez-Vazquez R, Ramponi R, Turri S, et al. (2012) Femtosecond laser microstructuring for polymeric lab-on-chips. J Biophotonics 5: 687702. [Crossref]
45. von der Linde D, Sokolowski-Tinten K, Bialkowski J (1997) Laser-solid interaction in the femtosecond time regime. Appl Surf Sci 109-110: 1-10.

46. Sahler R, Bille JF, Enright S, Chhoeung S, Chan K (2016) Creation of a refractive lens within an existing intraocular lens using a femtosecond laser. J Cataract Refract Surg 42: 1207-1215. [Crossref]

47. Huxlin Lab - University of Rochester Medical Center. LIRIC - a new paradigm in refractive error correction n.d. https://www.urmc.rochester.edu/eye-institute/research labs/huxlin/projects/liric.aspx (accessed October 27, 2019).

48. Zheleznyak L. Ophthalmology/Femtosecond Lasers: LIRIC: Next-generation refractive laser surgery 2016. https://www.bioopticsworld.com/biomedicine/article/16429510/ ophthalmologyfemtosecond-lasers-liric-nextgeneration-refractive-laser-surgery (accessed October 26, 2019).

49. Ding L. Micro-processing of polymers and biological materials using high repetition rate femtosecond laser pulses. University of Rochester, 2009.

Copyright: $\odot 2020$ Kareliotis G. This is an open-access article distributed under the terms of the Creative Commons Attribution License, which permits unrestricted use, distribution, and reproduction in any medium, provided the original author and source are credited. 